\title{
Evaluation of the Pinus taeda quality using ultrasound
}

\author{
Ozana Maria de Andrade Maia \\ CPGEI \\ Federal University of Technology - Paraná (UTFPR) \\ Curitiba, Paraná, Brazil \\ ozanamaia@utfpr.edu.br \\ Marcelo Real Prado \\ DAQBI \\ Federal University of Technology - Paraná (UTFPR) \\ Curitiba, Paraná, Brazil
}

\begin{abstract}
The pinus is a type of wood that is widely used as feedstock for the production of softwood pulp and higher quality paper, boards, MDF (Medium Density Fiberboard), OSB (Oriented Standard Board), plywood, furniture and others. The main problem for the pinus is the attack by the wood wasps (Sirex noctilio F., 1793) (Hymenoptera; Siricidae) that damages the wood due to galleries built by the wasps larvae. The use of nondestructive techniques to assess the quality and to evaluate mechanically the wood has been the focus of many studies and is certainly an essential tool to decrease the production costs. This work aimed to evaluate the damage due to the infestation of $S$. noctilio in Pinus taeda $\mathbf{L}$. using ultrasound. The tests have been carried out in the through transmission mode, using a pulserreceiver, two pairs of ultrasound transducers $(50 \mathrm{kHz}$ and 100 $\mathrm{kHz}$ of central frequencies for each), a digital oscilloscope and a portable microcomputer for data storage. The initial tests have been done using seven logs of pinus samples (lengths in the range $0.70-0.94 \mathrm{~m}$ and diameters $0.09-0.18 \mathrm{~m}$ ). The transducers were positioned in the samples to measure the ultrasound propagation velocity and the attenuation in the axial, radial (lower, middle and upper parts of the logs), longitudinal (middle and upper parts), inclined at approximately 45 degrees (middle and upper parts), longitudinal/transversal (middle and upper parts) and inclined/transversal (middle and upper parts) modes. The initial results of the 960 tests (430 with each pair of transducers) that have been done with the pinus samples have shown that the infested samples presented a decrease in the propagation velocity and an increase in the attenuation when compared to the control sample results. The initial results showed that the ultrasound technique can help the quality evaluation of $P$. taeda and to detect the infestation of wood wasps. Further tests should be made with a larger number of samples to evaluate the elastic modulus and other ultrasound parameters. The results will be used to define a novel test protocol to be used for material characterization in the agronomical area.
\end{abstract}

Keywords- ultrasound; material characterization; wood quality; pinus.

\footnotetext{
Brazilian Sponsoring Agencies: Coordenação de Aperfeiçoamento de
Pessoal de Nível Superior (CAPES), Fundação Araucária de Apoio ao

Brazilian Sponsoring Agencies: Coordenação de Aperfeiçoamento de
Pessoal de Nível Superior (CAPES), Fundação Araucária de Apoio ao Desenvolvimento Científico e Tecnológico do Estado do Paraná and Conselho Nacional de Desenvolvimento Científico e Tecnológico (CNPq).
}

\author{
Fabio Kurt Schneider, Joaquim Miguel Maia, Mayara \\ Fernanda Gimenes de Souza \\ DAELN/CPGEI \\ Federal University of Technology - Paraná (UTFPR) \\ Curitiba, Paraná, Brazil
}

\author{
Susete do Rocio Chiarello Penteado, Wilson Reis \\ Filho, Edson Tadeu Iede \\ Brazilian Agricultural Research Corporation - EMBRAPA \\ Colombo, Paraná, Brazil
}

\section{INTRODUCTION}

Currently, due to the low cost of production, the pinus is one of the most used types of wood as a raw material in the construction industry. It is used as feedstock for the production of softwood pulp and higher quality paper, boards, MDF (Medium Density Fiberboard), OSB (Oriented Standard Board), plywood, laminates, furniture and others [1].

The occurrence of new pests in Pinus spp. in Brazil and other countries has grown substantially due to the fact that, in recent years, attacks by the introduced species and also by the adaptation of native species have been recorded. Factors such as the lack of specific natural enemies, plenty of food available and monoculture have favored an increase in population of these pests and, consequently, increasing the production costs [2].

The main pest of pinus reforestation is the wood wasps (Sirex noctilio F., 1793) (Hymenoptera; Siricidae), species originated in Europe, Asia and North Africa [3]. The main symptoms of the attack by the wood wasp are [2]: 1) resin spills arising from punctures made by the females to lay their eggs and, in some cases, it is observed runoff of the resin; 2) yellowing of the treetop after the attack, with a yellowish at an early stage, after it became reddish-brown and dry, until the fall of the needles; 3) emergence of holes in the wood bark, where the adults wasps leave the plant; 4) bluish spots caused by a secondary fungus belonging to the genus Botryodiplodia; 5) galleries inside the timber, built by the larvae.

The use of nondestructive techniques to assess the quality of the wood have been the focus of many studies [4], [5], [6]. This work aimed to evaluate the damage due to the infestation of S. noctilio in Pinus taeda L. using the ultrasound technique. 


\section{MATERIAL AND METHODS}

The setup used to evaluate the $P$. taeda samples is formed by a 5077PR pulser-receiver (Olympus Corporation), a pair of ultrasound transducers with $50 \mathrm{kHz}$ of central frequencies (models 083-067-038, GE Inspection Technologies), a pair of transducers with $100 \mathrm{kHz}$ central frequencies (models 083-058769, GE Inspection Technologies), a MSO4104B digital oscilloscope (Tektronix Inc.) and a portable microcomputer for data storage.

The pulser-receiver was used to excite the transmitting transducer with negative pulses $(-400 \mathrm{~V})$ and to condition the ultrasonic waves that propagated through the pinus samples and were received by the receiver transducer. The received ultrasound signals from the $50 \mathrm{kHz}$ and the $100 \mathrm{kHz}$ transducers were amplified by $20 \mathrm{~dB}$ and $40 \mathrm{~dB}$, respectively. The conditioned signals were acquired by the digital oscilloscope and transferred via USB interface to the computer to be processed.

Each pair of transducers (Transmitter - $\mathrm{T}$ and a Receiver R) was positioned (Fig. 1) in the samples to measure the ultrasound propagation velocity and the attenuation in the following modes:

- Axial (Fig. 1a).

- Radial (Fig. 1b), with the pair of transducers positioned at the lower (I), middle (M) and upper (S) parts of the logs. The distance between I and $\mathrm{M}$ was $0.25 \mathrm{~m}$ and between I and $\mathrm{S}$ was $0.50 \mathrm{~m}$.

- Longitudinal (Fig 1c), with the transducers positioned orthogonally at the same side in the middle and upper parts of the log.

- Inclined at approximately 45 degrees (Fig 1d) and the receiver positioned first at the middle and later at upper parts of the log.

- Longitudinal/transversal (Fig 1e), with the receiver at the middle and upper parts.

- Inclined/transversal (Fig 1f), with the receiver at the middle and upper parts of the log.

The tests were carried out at the Ultrasound Laboratory of the Graduate School of Electrical Engineering \& Computer Science at the Federal University of Technology - Paraná (LUS/CPGEI/UTFPR) and at the Brazilian Agricultural Research Corporation - EMBRAPA, using seven short logs of $P$. taeda, with lengths in the range $0.70 \mathrm{~m}-0.94 \mathrm{~m}$ and diameters $0.09 \mathrm{~m}-0.18 \mathrm{~m}$. It was also tested a reference $\log$, without the attacks of the wood wasps.

The samples were prepared using a drill to make small holes where the transducers would be placed and it was used ultrasound transmission gel to couple them.

The ultrasound signals were acquired five times for each position of the pair of transducers, totalizing 960 tests (430 for each pair of transducers).

The acquired data was processed using the Matlab (Mathworks Inc.) and it was calculated the group longitudinal

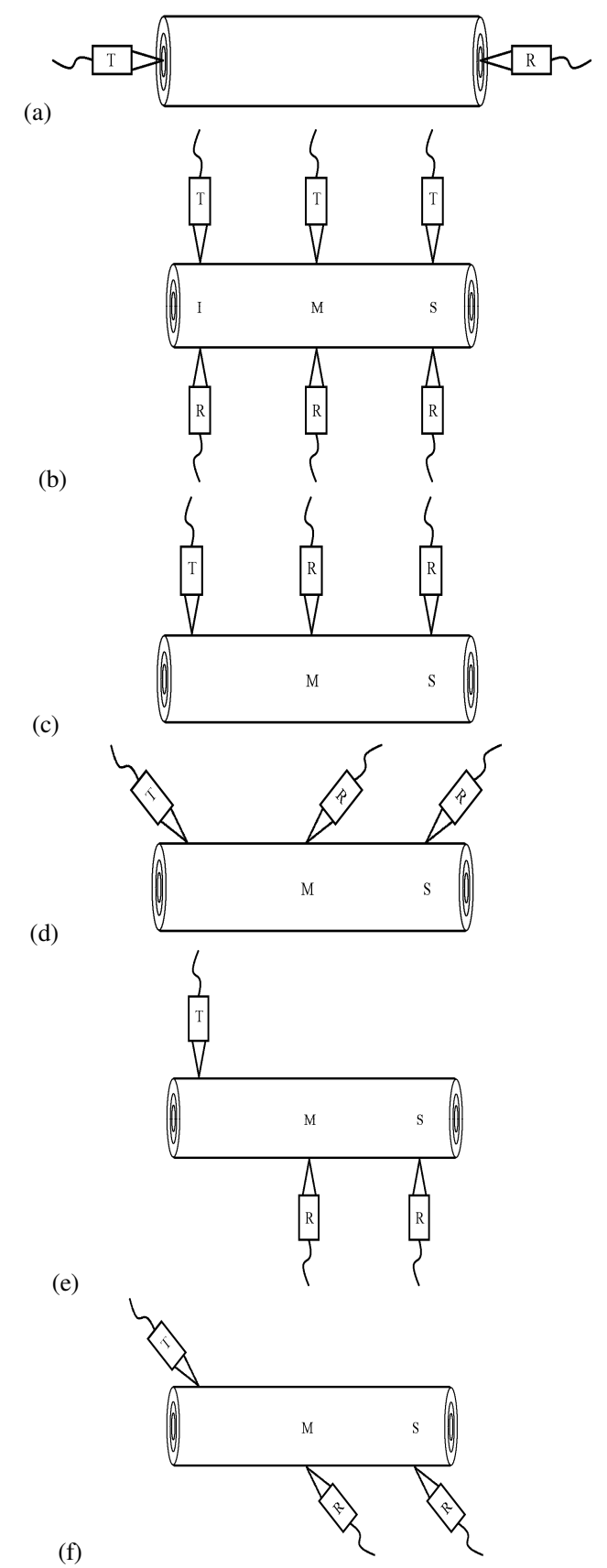

Figure 1. Positioning of the transmitter (T) and the receiver (R) ultrasound transducers to measure the attenuation and the velocity in the pinus samples: (a) axial mode; (b) radial mode in the lower (I), middle (M) and upper (S) parts of the logs; (c) longitudinal (M and S); (d) inclined at approximately 45 degrees (M and $\mathrm{S}$ ); (e) longitudinal/transversal (M and $\mathrm{S}$ ) and (f) inclined/transversal (M and $\mathrm{S})$.

propagation velocities and the attenuation for the $100 \mathrm{kHz}$ and $50 \mathrm{kHz}$ transducers signals.

\section{RESULTS AND DISCUSSION}

The equipment used for testing and an example of positioning the transducers on the sample for the axial mode are shown in Fig. 2a and Fig. 2b., respectively. 


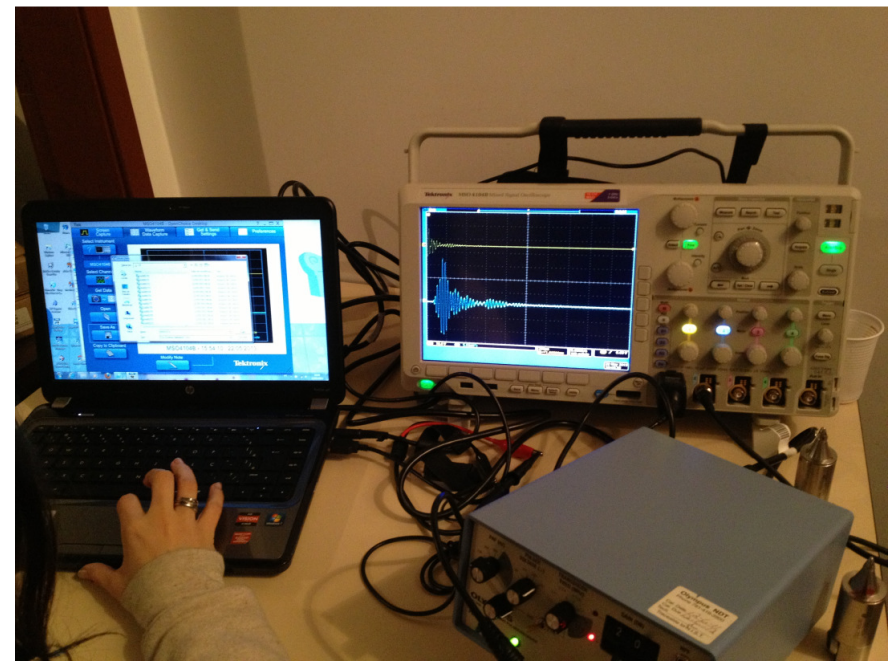

(a)

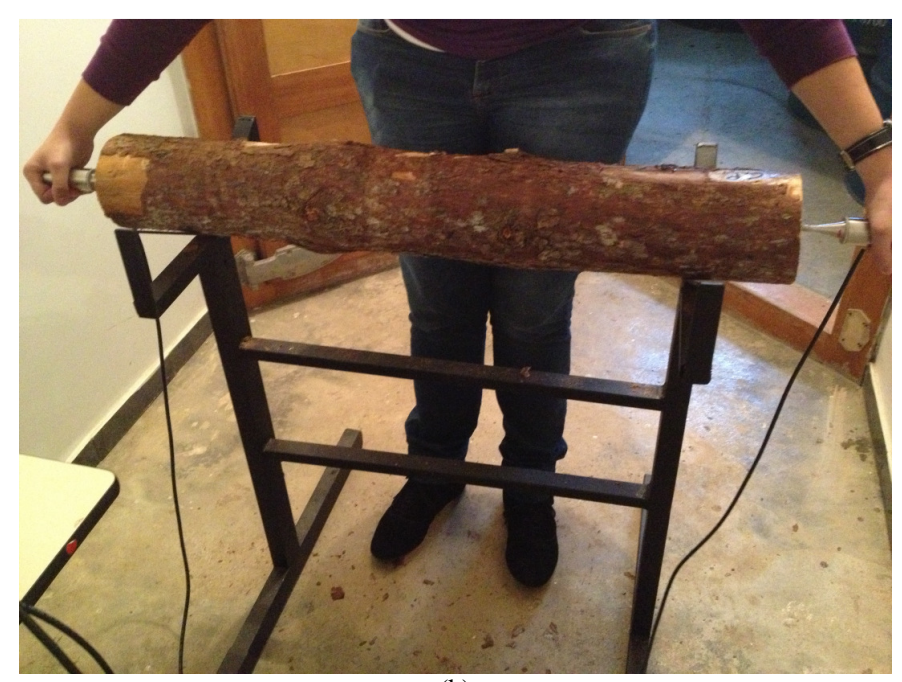

(b)

Figure 2. (a) Equipment used to perform the tests and (b) an example of positioning of the transducers to acquire data in the axial mode.

Fig. 3 shows the number of signs of attack (holes in the bark) by the wood wasps detected in each sample evaluated and the mean attenuation values between the signals received by the $50 \mathrm{kHz}$ and the $100 \mathrm{kHz}$ central frequencies ultrasound receivers. The mean values of the attenuation were calculated using the five signals received by each transducer $(50 \mathrm{kHz}$ and $100 \mathrm{kHz}$ ). Using as a basis Fig. 2, that shows the positioning of the transducers for each test, the attenuations presented in Fig. 3 were calculated for the signals received in the axial mode (Fig. 3a) and radial mode at lower (Fig. 3b) parts of the logs.

Fig. 3c, Fig. 3d, Fig. 3e and Fig. 3f, shows the results obtained for the tests with the receivers positioned at the upper parts of the logs for longitudinal (ALS), inclined (AIS), longitudinal/transversal (ALST) and inclined/transversal (AIST) modes, respectively. The results obtained with the transducers positioned at the middle parts of the logs were calculated and they are similar to those presented in Fig. 3.

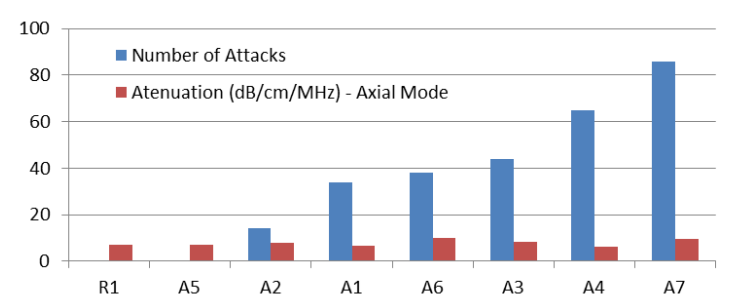

(a)

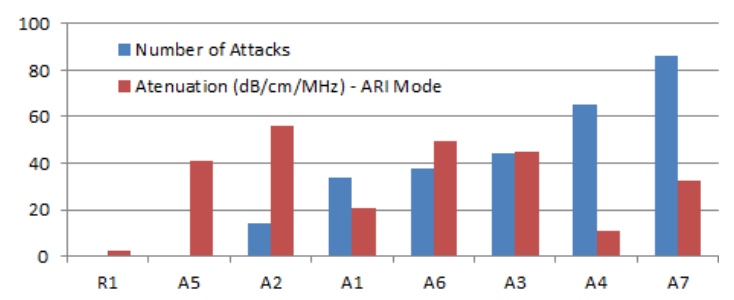

(b)

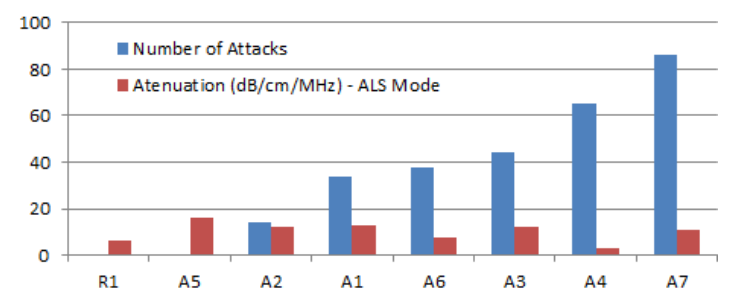

(c)

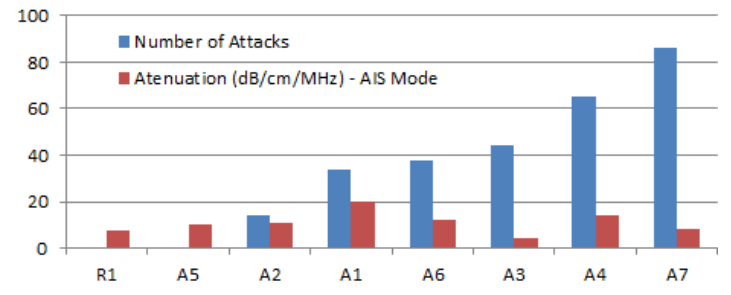

(d)

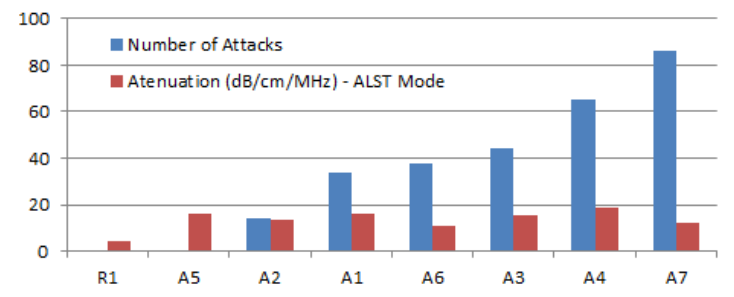

(e)

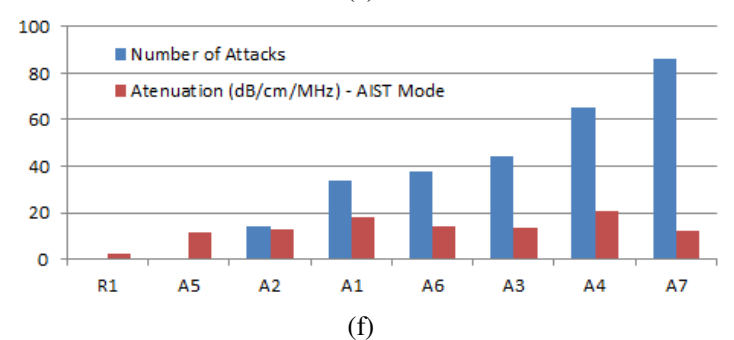

Figure 3. Number of holes (signs of attack by the wood wasps) detected in each sample evaluated and the attenuation measured between the transducers of $50 \mathrm{kHz}$ and $100 \mathrm{kHz}$, for the tests performed in the (a) axial mode; (b) radial mode in the lower (ARI) parts of the logs; (c) longitudinal (ALS), (d) inclined (AIS), (e) longitudinal/transversal (ALST) and (f) inclined/transversal (AIST) at the upper parts of the logs. 
Comparing the results of the attenuations obtained for samples A1, A2, A3, A4, A6 and A7, with signs of attack by wood wasps, it is possible to see that most of them have values higher than the attenuation of the reference sample (R1). The exception occurred for sample A5, which has no signs of attack by the wood wasps, but also provides high attenuation. This was due to the presence of wood knots, which attenuate the ultrasonic waves. The same happened for other samples which had lower attenuation than the reference in some modes of test.

The propagation velocities of ultrasonic waves were measured in all modes of placement of the transducers for each sample. However, the mode with the highest difference in the velocities measured in samples attacked by the wood wasps and the reference samples was the axial. This may be due to the short lengths of samples evaluated in this work and the presence of knots in some modes of test. The values for the velocities measured in the axial mode are presented in Table I.

TABLE I. GROUP VELOCITIES OF THE ULTRASONIC WAVES MEASURED FOR EACH SAMPLE IN THE AXIAL MODE.

\begin{tabular}{|l|c|c|}
\hline \multirow{2}{*}{ Sample } & \multicolumn{2}{|c|}{ Group Velocity \pm Standard Deviation $(\mathbf{m} / \mathbf{s})$} \\
\cline { 2 - 3 } R1 & $\boldsymbol{V}$ & $\boldsymbol{S}$ \\
\hline A1 & 3033.5 & 3.1 \\
\hline A2 & 2672.7 & 4.6 \\
\hline A3 & 2431.8 & 12.7 \\
\hline A4 & 2736.3 & 30.7 \\
\hline A5 & 2606.3 & 76.0 \\
\hline A6 & 2613.1 & 34.5 \\
\hline A7 & 2564.9 & 17.5 \\
\hline
\end{tabular}

Some studies have been done using samples larger and also presenting higher variations than those obtained in this work [7][8]. The difference may be due the fact that in some works they do not separate the longitudinal from the shear wave's velocities, using the point of maximum amplitude as a trigger for the measurements. This can lead to errors, because sometimes the first signal peaks, related to a longitudinal wave, are smaller than the last, which may be due a shear mode that has lower velocities than the longitudinal one or due to reflections on the edge of the log.

Fig. 4 shows the signals acquired from the same sample, in the axial mode, where it is possible to see that the first maximum envelope peak lead to a velocity of $2600 \mathrm{~m} / \mathrm{s}$ for the signal presented in Fig. $4 \mathrm{a}$ and $1474 \mathrm{~m} / \mathrm{s}$ for the signal presented in Fig. 4b.

\section{CONCLUSION}

The initial results of the tests that have been done with the samples of pinus have shown that the samples attacked by the wood wasps presented a decrease in the propagation velocity and an increase in the attenuation when compared to the control sample results. These initial results showed that the ultrasound technique can support the quality evaluation of $P$. taeda and detection of infestation of wood wasps.

Further tests should be made with a larger number of samples to evaluate the elastic modulus and other ultrasound parameters. The results will be used to define a novel test protocol to be used for material characterization in the agronomical area.

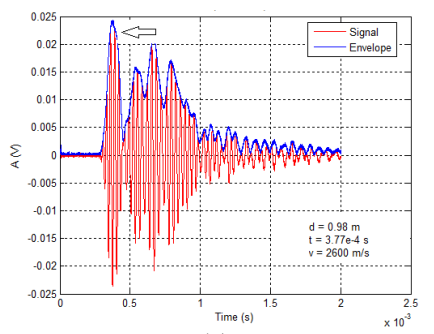

(a)

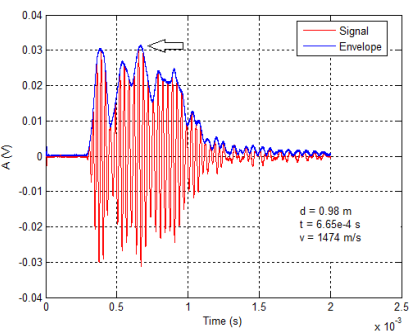

(b)
Figure 4. Signals obtained from the same sample, showing that the use of the point of maximum amplitude in the envelope may lead to erros in the velocities calculations. The fisrt peak is related to a longitudinal mode and the other to shear modes or to reflections in the log edge. The arrows indicate the point for which the velocities were calculated: (a) $v=2600 \mathrm{~m} / \mathrm{s}$; (b) $\mathrm{v}=1474 \mathrm{~m} / \mathrm{s}$.

\section{ACKNOWLEDGMENT}

The authors thank Coordenação de Aperfeiçoamento de Pessoal de Nível Superior (CAPES), Fundação Araucária de Apoio ao Desenvolvimento Científico e Tecnológico do Estado do Paraná and Conselho Nacional de Desenvolvimento Científico e Tecnológico $(\mathrm{CNPq})$ for the financial support.

\section{REFERENCES}

[1] C. G. Auer, A. G. Junior, A. F. Santos, "Cultivo do Pinus. Sistemas de Produção", Florestas Versão Eletrônica, n. 5, nov. 2005. Available in:http://sistemasdeproducao.cnptia.embrapa.br/FontesHTML/Pinus/Cult ivodoPinus. Accessed in 08/04/2011.

[2] S. R. C. Penteado, E. T. Iede, W. Reis Filho, "Manual para o controle da vespa-da-madeira em plantios de pinus", Colombo: Embrapa Florestas, 2002. 38 p.

[3] E. T. Iede, S. R. C. Penteado, J. C. Bisol, "Primeiro registro de ataque de Sirex noctilio em Pinus taeda no Brasil", Colombo: Embrapa-CNPF, 1988. $12 \mathrm{p}$.

[4] J. L. Sandoz, Y. Benoit, "Non-destructive evaluation for new wooden poles quality assessment", CBS-CBT, 2011, Available in: http://www. sylvatest.com/. Accessed in: 08/02/2011.

[5] V. Tanasoiu, C. Miclea, C. Tanasoiu, "Nondestructive testing techniques and piezoelectric ultrasonics transducers for wood and built in wooden structures", Journal of Optoelectronics and Advanced Materials, v.4, n. 4, p. $949-957,2002$.

[6] V. Giurgiutiu, A. N. Zagrai, "Embedded self-sensing piezoelectric active sensors for on-line structural identification", Jounal of Vibration Acoustics, vol. 124, pp. 116- 125, 2002.

[7] V. Bucur, “Acoustics of wood”, 2.ed.New York: Springer-Verlag, 2006. 393p.

[8] A. Bartholomeu, R. Gonçalves, V. Bucur, "Dispersion of ultrasonic waves in Eucalyptus lumber as a function of the geometry of boards", Scientia Forestalis, n.63, p.235-240, 2003. 